\title{
A Finite Volume Method Solution for the Bidomain \\ Equations and their Application to Modelling Cardiac \\ Ischaemia
}

Peter R. Johnston

School of Biomolecular and Physical Sciences,

Griffith University,

Nathan,

Queensland,

Australia, 4111

Phone: 61-7-3735-7748,

Fax: 61-7-3735-7656,

E-mail: P.Johnston@griffith.edu.au

May 18, 2009 


\begin{abstract}
This paper presents an implementation of the finite volume method with the aim of studying subendocardial ischaemia during the ST segment. In this implementation, based on hexahedral finite volumes, each quadrilateral sub-face is split into two triangles to improve the accuracy of the numerical integration in complex geometries and when fibre rotation is included.

The numerical method is validated against previously published solutions obtained from slab and cylindrical models of the left ventricle with subendocardial ischaemia and no fibre rotation. Epicardial potential distributions are then obtained for a half-ellipsoid model of the left ventricle. In this case it is shown that for isotropic cardiac tissue the degree of subendocardial ischaemia does not affect the epicardial potential distribution, which is consistent with previous findings from analytical studies in simpler geometries.

The paper also considers the behaviour of various preconditioners for solving numerically the resulting system of algebraic equations resulting from the implementation of the finite volume method. It is observed that each geometry considered has its own optimal preconditioner.
\end{abstract}

Keywords: Bidomain equations, numerical solutions, electrocardiography, finite volume method, ischaemic tissue. 


\section{Introduction}

Solving the bidomain [1] equations [2, 3] has become the predominant method of obtaining insight into the electrical activity of the human heart. These solution methods are generally numerical in nature and are based on the finite difference method $[4,5,6]$, the finite element method $[7,8,9,10]$ and the finite volume method $[11,12]$. Most of these studies consider propagation of the action potential through various two and three dimensional shapes of healthy cardiac tissue. However, the bidomain equations have also been used to study subendocardial ischaemia during the ST segment $[13,14,15]$. The ST segment being the isoelectric portion of the electrocardiogram between the QRS complex, where the ventricles of the heart depolarise, and the $\mathrm{T}$ wave, where the ventricles repolarise [16]. At the cellular level, this interval corresponds to the plateau phase of the action potential [17] where each cell is at the same potential, thus giving rise to the isoelectric status of the tissue.

The modelling of cardiac ischaemia, both subendocardial and transmural, is important in understanding how it relates to coronary heart disease and its predictive value for sudden cardiac death. A recent excellent review [18] highlights many of the benefits and limitations of modelling in this area. This situation has also been the focus of experimental studies $[19,20]$ and direct numerical simulation $[21]$.

Previous studies of the effect of subendocardial ischaemia during the ST segment have been limited to simple geometries $[13,14,15]$. Due to the simple geometries considered, solutions could be obtained using analytical techniques with only minimal augmentation by numerical methods. This is a limitation of these studies, so to consider more realistic geometries, a full numerical method is required.

In this paper, an implementation of the finite volume method is presented with the aim of studying the effect of subendocardial ischaemia during the ST segment. The implementation is based on 
that described by Penland et. al [11], but is extended by subdividing quadrilateral control volume sub-faces into triangles to better account for cardiac fibre rotation and to better approximate more complex geometries. The bidomain equations are solved in a steady state situation, reflecting the static behaviour during the ST segment [16].

The first part of this paper describes in detail the construction of the finite volume method solution of the bidomain equations and highlights the differences from previous implementations. Iterative solution strategies to solve the resulting system of algebraic equations are then discussed. The finite volume method solution is then validated in both slab and cylindrical geometries by comparing with solutions obtained previously $[13,14]$. Next, solutions are obtained in an ellipsoidally shaped approximation to the left ventricle, where it is verified that the degree of subendocardial ischaemia does not affect the epicardial potential distribution in an isotropic model. Finally, there is a comparison of the benefits of several iterative solution techniques, including timing and convergence issues.

\section{Methods}

\subsection{The Bidomain Model}

The bidomain model [1] for cardiac tissue, or myocardium, provides a continuum approximation for the electrical properties of the tissue based on electrical behaviour at the cellular level. Since the ST segment is assumed to be isoelectric and each cell is in its plateau phase at the same potential, a steady state model can be assumed to govern the intracellular potential, $\phi_{i}$, and the extracellular potential, $\phi_{e}$, in the tissue:

$$
\nabla \cdot\left(\mathbf{M}_{i} \nabla \phi_{i}\right)=I_{m}
$$


and

$$
\nabla \cdot\left(\mathbf{M}_{e} \nabla \phi_{e}\right)=-I_{m}
$$

where $\mathbf{M}_{i}$ and $\mathbf{M}_{e}$ are the intracellular $(i)$ and extracellular $(e)$ conductivity tensors, respectively, and $I_{m}$ is the membrane current. The equations (1) and (2) can be obtained from the vector form of Ohm's law [11]. The tensors $\mathbf{M}_{i}$ and $\mathbf{M}_{e}$ (represented via matrices) reflect the fact that the electrical conductivity of the cardiac tissue is anisotropic, since it is much easier for current to flow along the fibres than across them. In general six conductivities are required to describe the behaviour of the tissue, but here it is assumed that the conductivities in both transverse directions are equal. Hence, only four conductivities are required: intracellular $(i)$ and extracellular $(e)$ conductivities both along the fibres (longitudinal $(l)$ ) and across the fibres (transverse $(t)$ ). These conductivities are denoted by $\sigma_{i}^{l}, \sigma_{e}^{l}, \sigma_{i}^{t}$ and $\sigma_{e}^{t}$. Further, if the fibres rotate while moving through the tissue, the tensors also depend on the local fibre directions.

In the models to be considered here, the myocardium is assumed to be in contact with a blood cavity, which can be considered to be an isotropic extracellular bath. In this region, the blood potential $\phi_{b}$ satisfies Laplace's equation

$$
\nabla^{2} \phi_{b}=0
$$

In order to facilitate the numerical treatment of these equations Roth [22] proposed a transformation of the equations in terms of the transmembrane potential $\phi_{m}=\phi_{i}-\phi_{e}$. Adding equations (1) and (2), with some algebra gives

$$
\nabla \cdot\left(\mathbf{M}_{i}+\mathbf{M}_{e}\right) \nabla \phi_{e}=-\nabla \cdot\left(\mathbf{M}_{i} \nabla \phi_{m}\right)
$$

This equation is known as the bidomain passive current flow equation [23, 17] and is routinely used to model current flow during the ST segment. As will be discussed later, $\phi_{m}$ will be assumed to be a known function of position. 


\subsection{Boundary Conditions}

Typical boundary conditions for the above equations arise at the tissue-air, tissue-blood and bloodair interfaces. Krassowska and Neu [24] undertook a formal study of the boundary conditions relating to the bidomain equations and have suggested the following. At tissue-air interfaces, no current flux is assumed at the boundary of the intracellular and extracellular domains, that is

$$
\mathbf{M}_{i} \nabla \phi_{i} \cdot \mathbf{n}=0
$$

and

$$
\mathbf{M}_{e} \nabla \phi_{e} \cdot \mathbf{n}=0
$$

where $\mathbf{n}$ is the outward pointing normal vector at the boundary. At the blood-air interfaces there is a similar condition

$$
\sigma_{b} \nabla \phi_{b} \cdot \mathbf{n}=0
$$

where $\sigma_{b}$ is the conductivity of the blood. However, at least one node on the blood-air interface must be grounded to achieve a unique solution of the governing equations and provide a reference potential for the system, i.e.,

$$
\left.\phi_{b}\right|_{\text {ground }}=0
$$

At the blood-tissue interface the extracellular potential must equal the blood potential. Also, the intracellular current density normal to the boundary must remain in the tissue and the extracellular current density normal to the boundary must flow into the blood. In other words, there must be continuity of potential and current in the extracellular spaces across this boundary. These conditions 
can be expressed as

$$
\begin{aligned}
\phi_{e} & =\phi_{b} \\
\mathbf{M}_{e} \nabla \phi_{e} \cdot \mathbf{n} & =\sigma_{b} \nabla \phi_{b} \cdot \mathbf{n} \\
\mathbf{M}_{i} \nabla \phi_{i} \cdot \mathbf{n} & =0
\end{aligned}
$$

where $\mathbf{n}$ is the outward normal vector from the tissue.

\subsection{Conductivity Tensor}

The nature of cardiac tissue is such that the cells within the myocardium align themselves in sheets of parallel fibres [25]. As a result of this arrangement, it is easier for current to flow along the fibres than it is across them or between sheets, both in the intracellular and extracellular spaces. For fibres which rotate in three dimensional space, define a local coordinate system with orthogonal basis vectors given in the global coordinate system as $\mathbf{a}_{l}$ (along-fibre or longitudinal), $\mathbf{a}_{t}$ (cross-fibre or transverse) and $\mathbf{a}_{s}$ (cross-sheet) [16]. Further, let the conductivities in the corresponding directions in the intracellular and extracellular spaces be $\sigma_{q}^{l}, \sigma_{q}^{t}$ and $\sigma_{q}^{s}$ (where $q=i$ or $e$ ). Then, in the local coordinate system, the conductivity tensor can be expressed as a diagonal matrix

$$
\boldsymbol{\Sigma}_{\mathbf{q}}=\left(\begin{array}{ccc}
\sigma_{q}^{l} & 0 & 0 \\
0 & \sigma_{l}^{t} & 0 \\
0 & 0 & \sigma_{q}^{s}
\end{array}\right) .
$$

The matrix $\mathbf{U}$ defined by $\mathbf{U}=\left(\mathbf{a}_{l}, \mathbf{a}_{t}, \mathbf{a}_{s}\right)$, is a $3 \times 3$ rotation matrix mapping the local fibre direction into the global three dimensional coordinate system. This then leads to expressions for the conductivity tensors $\mathbf{M}_{i}$ and $\mathbf{M}_{e}$ as

$$
\mathbf{M}_{q}=\mathbf{U} \Sigma_{q} \mathbf{U}^{T}
$$

for $q=i$ or $e$. 
It is generally assumed, as will be the case here, that the cross-fibre and cross-sheet conductivities are equal, resulting in the necessity for only four conductivity parameters $\sigma_{i}^{l}, \sigma_{e}^{l}, \sigma_{i}^{t}$ and $\sigma_{e}^{t}$. Further assumptions are that the sheets run parallel to the epicardium and that the fibre directions of each sheet rotate through a given angle $\alpha$, varying linearly with depth.

\subsection{Ischaemia Model}

To describe the region of ischaemia, consider a local coordinate system, $\left(\tau_{1}, \tau_{2}, n\right)$ where $n$ is the perpendicular direction leading from the endocardium to the epicardium and $\tau_{1}$ and $\tau_{2}$ are two orthogonal directions, mutually orthogonal to $n$. It will be assumed that the ischaemic tissue occupies a finite region in the $\tau_{1}$ and $\tau_{2}$ directions. In the $n$ direction the region is bounded by the endocardium but does not extend to the epicardium (as this would then be full thickness ischaemia). Hence, the ischaemic region can be described as

$$
\mathcal{I}=\left\{\left(\tau_{1}, \tau_{2}, n\right) \mid-a_{\tau_{1}} \leq \tau_{1} \leq a_{\tau_{1}},-a_{\tau_{2}} \leq \tau_{2} \leq a_{\tau_{2}}, 0 \leq n \leq a_{n}\right\}
$$

However, as will be discussed shortly, the region does not necessarily have a sharp boundary, as indicated by the set $\mathcal{I}$. If the presence of a so-called ischaemic boundary is assumed, then there is a smooth transition between ischaemic and normal tissue. In this case, the quantities $a_{\tau_{1}}, a_{\tau_{2}}$ and $a_{n}$ represent the centres of the ischaemic boundary.

To specify an analytic representation for the ischaemic region, the product representation for the transmembrane potential distribution

$$
\phi_{m}\left(\tau_{1}, \tau_{2}, n\right)=\Delta \phi_{p} \Psi\left(\tau_{1}\right) \Psi\left(\tau_{2}\right) \Psi(n),
$$

suggested by Tung [2] is employed. Here $\Delta \phi_{p}$ is the difference in plateau potentials between normal 
and ischaemic tissue. In any particular direction, $t$, the shape function, $\Psi(t)$, is defined by

$$
\Psi(t)= \begin{cases}\frac{1-e^{-a_{t} / \lambda_{t}} \cosh t / \lambda_{t}}{1-e^{-a_{t} / \lambda_{t}}} & |t| \leq a_{t} \\ \frac{e^{-|t| / \lambda_{t}} \sinh a_{t} / \lambda_{t}}{1-e^{-a_{t} / \lambda_{t}}} & |t|>a_{t}\end{cases}
$$

where $t$ is $\tau_{1}, \tau_{2}$ or $n$. The parameters $\lambda_{t},\left(t=\tau_{1}, \tau_{2}, n\right)$ govern the width of the ischaemic boundary and will be discussed in Section 2.7. Figure 1 shows a plot of the function $\Psi(t)$ with $\lambda_{t}$ values of 0.01 and 0.1 . Note that the smaller the value of $\lambda_{t}$, the more rapid the change from ischaemic to normal tissue.

\subsection{Mesh}

In this paper three distinct meshes will be considered. The first two, a slab mesh and a cylindrical mesh, are included as part of the validation process, to compare with previously published models in these geometries $[13,14,26]$. The third mesh is a half-ellipsoid mesh $[7,11]$ which is used to represent a simplified left ventricle.

The slab model is constructed in a rectilinear $(x, y, z)$ coordinate system. The epicardial surface is the $x y(z=0)$ plane and the endocardial surface is the $z=1$ plane defined over the region $-x_{L} \leq x \leq x_{L}$ and $-y_{L} \leq y \leq y_{L}$. (Note that the $z$ direction in this model is defined in the opposite sense to the $n$ direction defined in the previous section.) A blood mass, in contact with the endocardium, extends to a depth $z_{D}$ in the positive $z$-direction. There are $x_{N}$ nodes in the $x$-direction and $y_{N}$ nodes in the $y$-direction. Across the tissue there are $z_{T}$ nodes in the $z$-direction and the blood mass consists of $z_{B}$ nodes, also in the $z$-direction.

As mentioned above there is an ischaemic region within the tissue, covering the region $-x_{i} \leq$ $x \leq x_{i},-y_{i} \leq y \leq y_{i}, z_{i} \leq z \leq 1$. To account for the high current densities likely to occur across the ischaemic boundaries, nodes are concentrated near these boundaries. Node clustering is achieved 
using a sinh transformation [27] of the form

$$
s=a+b \sinh (\mu t-\eta)
$$

where

$$
\mu=\frac{1}{2}\left\{\operatorname{arcsinh}\left(\frac{1+a}{b}\right)+\operatorname{arcsinh}\left(\frac{1-a}{b}\right)\right\}
$$

and

$$
\eta=\frac{1}{2}\left\{\operatorname{arcsinh}\left(\frac{1+a}{b}\right)-\operatorname{arcsinh}\left(\frac{1-a}{b}\right)\right\}
$$

In this transformation, the parameter $b$ controls the degree of clustering of the nodes. After experimentation it was found that the best choice for $b$ was 0.1 . The parameter $a$ specifies the point around which the nodes should be clustered. The transformation given by (17), (18) and (19) is a mapping from $[-1,1]$ onto $[-1,1]$ with $-1 \leq a \leq 1$. This transformation must in turn be transformed to account for the size of the domain in the various coordinate directions. Figure 2 demonstrates how mesh points are clustered around the point $s=0$ (ie $a=0)$ for different values of $b(b=1, b=0.1$ and $b=0.01$ ). Setting $b=1$ is equivalent to no clustering of the points, and decreasing the value of $b$ shows greater clustering of the mesh points.

For the cylindrical model of the left ventricle, the mesh was created from a typical butterfly mesh (Figure 3(a)) in the $x y$ plane and extruded along the $z$-axis. The inner square of the mesh was mapped into a circle which becomes the blood cavity and the four "wings" of the mesh were mapped into an annular region which became the ventricular muscle region. Mesh clustering was utilised in the radial direction at the top of the ischaemic region and in the $z$-direction, at the lateral boundaries. Figure 3(b) shows the mesh with 23 nodes along the sides of the inner square and 30 nodes through the ventricular muscle.

For the ellipsoid model of the left ventricle the mesh for the tissue was created in a similar fashion to that for the cylindrical mesh. A circular mesh of radius $R$ was created in the $x y$-plane with a 
butterfly mesh. The inner nodes with radius less than $R$ were displaced in the $z$-direction to fit onto the surface of a half ellipsoid given by

$$
\begin{array}{ll}
x=a \cos \theta \cos \phi & -\pi \leq \phi \leq \pi \\
y=b \cos \theta \sin \phi & 0 \leq \theta \leq \frac{\pi}{2} \\
z=c \sin \theta &
\end{array}
$$

with the nodes of the circle of radius $R$ remaining in the $x y$ plane. The constants $a, b$ and $c$ govern the overall shape of the ellipsoid. Finally, the three dimensional tissue region was created by extruding the above surface mesh, in the direction normal to the surface, a distance equal to the thickness of the tissue. The blood cavity mesh was created by extruding a circular mesh in the $x y$ plane to fit inside the tissue mesh. For this mesh, node clustering was used only in the radial direction through the tissue.

\subsection{Finite Volume Method}

The finite volume method is the solution methodology of choice for this problem as it naturally handles the no flux boundary conditions used in this problem. The formal approach to be followed here closely mimics the formulation presented by Penland et al. [11] with a few modifications.

In contrast to the methods described by Penland et al. [11], here equations (4) and (3) will be solved on a mesh of unstructured hexahedral elements, constructed as described above, in three spatial dimensions. The meshes consist of a set of $n$ points $\mathbf{P}=\left\{P_{1}, \ldots, P_{n}\right\}$ with a set of $m$ elements $\mathbf{E}=\left\{E_{1}, \ldots, E_{m}\right\}$. As with all numerical solution procedures, approximations of the solution at the node points are required. Hence, let $\Phi_{m}$ and $\Phi_{e}$ be vectors whose elements $\left(\Phi_{m}\right)_{j}$ and $\left(\Phi_{e}\right)_{j}$, respectively, are the approximations to the solutions $\phi_{m}$ and $\phi_{e}$ at the node point $P_{j}$.

The finite volume method requires a set of $n$ boxes (or control volumes) $\mathbf{B}=\left\{B_{1}, \ldots, B_{n}\right\}$, each surrounding a mesh point. In this study, the boxes will be hexahedral. Let the box $B_{j}$ surround 
the point $P_{j}$ and have boundary $\partial B_{j}$. Further, assume that the box $B_{j}$ is constructed as a union of hexahedral sub-boxes $B_{j, k}$ (Figure 5) for $k \in \mathcal{E}\left(P_{j}\right)$ with boundary $\partial B_{j, k}$ consisting of three quadrilateral faces within each element, $E_{k}$, where $\mathcal{E}\left(P_{j}\right)$ is the set of elements attached to node $P_{j}$. Generally, each node $P_{j}$ is surrounded by eight elements (ie $\left|\mathcal{E}\left(P_{j}\right)\right|=8$ ) and hence eight sub-boxes $B_{j, k}$. Within each element the three boundary faces making up $\partial B_{j, k}$ contribute to the calculation of the flux through the control volume $B_{j, k}$. Following Penland et al. [11] the face of each sub-box is constructed by connecting the centroids of the element $(c)$ with those of the edges $(e)$ and faces $(f)$.

The finite volume method is implemented by integrating the governing equations (4) and (3) over each box $B_{j}$. For the sake of brevity, only the implementation for equation (4) will be discussed. Integrating equation (4) over the box $B_{j}$ gives

$$
\int_{B_{j}} \nabla \cdot\left(\mathbf{M}_{i}+\mathbf{M}_{e}\right) \nabla \phi_{e} d V=-\int_{B_{j}} \nabla \cdot \mathbf{M}_{i} \nabla \phi_{m} d V
$$

Using the divergence theorem gives

$$
\oint_{\partial B_{j}} \mathbf{n}^{T}\left(\mathbf{M}_{i}+\mathbf{M}_{e}\right) \nabla \phi_{e} d S=-\oint_{\partial B_{j}} \mathbf{n}^{T} \mathbf{M}_{i} \nabla \phi_{m} d S
$$

The above flux integrals are evaluated as the sum of flux integrals of each face of $\partial B_{j}$, hence equation (24) can be written as

$$
\sum_{E_{k} \in \mathcal{E}\left(P_{j}\right)} \oint_{\partial B_{j, k}} \mathbf{n}^{T}\left(\mathbf{M}_{i}+\mathbf{M}_{e}\right) \nabla \phi_{e} d S=-\sum_{E_{k} \in \mathcal{E}\left(P_{j}\right)} \oint_{\partial B_{j, k}} \mathbf{n}^{T} \mathbf{M}_{i} \nabla \phi_{m} d S .
$$

The integrals are evaluated numerically in a natural coordinate system $(\xi, \eta, \zeta)$ which is related to the physical $(x, y, z)$ coordinate system via the Jacobian matrix $J$.

As the original mesh is defined in terms of arbitrary hexahedral elements, a set of eight basis functions which maps into a trilinear "brick" is required (Figure 4). Such basis functions are defined by

$$
N_{i}=\frac{1}{8}\left(1-\xi_{i} \xi\right)\left(1-\eta_{i} \eta\right)\left(1-\zeta_{i} \zeta\right)
$$


where $\xi_{i}, \eta_{i}$ and $\zeta_{i}$ are given in Table 1.

For each volume element $E_{k}$ a quantity $\phi$ may be expressed as a linear combination of the basis functions as

$$
\phi(x, y, z)=\sum_{i=1}^{8} \phi_{i} N_{i}(\xi, \eta, \zeta)
$$

where the coefficient $\phi_{i}$ for the $i^{\text {th }}$ basis function is the corresponding value of $\phi$ at vertex $i$. Similar expressions also hold for the coordinates $(x, y, z)$ within the volume element in terms of the coordinates of the node points $\left(x_{i}, y_{i}, z_{i}\right)$.

The Jacobian $J$ maps the natural coordinate space into the physical space via the relationship

$$
\left[\begin{array}{l}
\partial x \\
\partial y \\
\partial z
\end{array}\right]=J\left[\begin{array}{l}
\partial \xi \\
\partial \eta \\
\partial \zeta
\end{array}\right]
$$

where

$$
J=\left[\begin{array}{ccc}
\frac{\partial x}{\partial \xi} & \frac{\partial x}{\partial \eta} & \frac{\partial x}{\partial \zeta} \\
\frac{\partial y}{\partial \xi} & \frac{\partial y}{\partial \eta} & \frac{\partial y}{\partial \zeta} \\
\frac{\partial z}{\partial \xi} & \frac{\partial z}{\partial \eta} & \frac{\partial z}{\partial \zeta}
\end{array}\right]
$$

The derivatives in the Jacobian can be found by differentiating the coordinate expressions, viz.

$$
\frac{\partial x(\xi, \eta, \zeta)}{\partial \xi}=\sum_{i=1}^{N_{b}} x_{i} \frac{\partial N_{i}(\xi, \eta, \zeta)}{\partial \xi}
$$

The next step is the computation of the flux integrals

$$
\oint \mathbf{n}^{T} \mathbf{M} \nabla \phi d S
$$

for tensor $\mathbf{M}$ and some scalar $\phi$. At this point the approach taken here differs from that taken by Penland et al. [11]. Penland et al. assumed that the faces $\partial B_{j, k}$ were flat, which is a reasonable assumption for a rectilinear grid and where there is minimal fibre rotation [12]. However, computations 
performed on the elliptical ventricular mesh showed that some triangular sub-faces of the $\partial B_{j, k}$ had normals which differed in direction up to $30^{\circ}$. Based on this observation and the fact that complex fibre rotations would be considered, each of the three faces comprising $\partial B_{j, k}$ was decomposed into two triangular sub-faces with normals $\mathbf{n}_{1}$ and $\mathbf{n}_{2}$ (Figure 5). Hence the contribution to the flux through the control volume $B_{j}$ from the element $E_{k}$ is calculated by integration over six triangular sub-faces of $\partial B_{j, k}$.

Assuming that the current density is constant on each face, then

$$
\oint_{\partial B_{j, k}} \mathbf{n}^{T} \mathbf{M} \nabla \phi d S=\sum_{l=1}^{6} \mathbf{n}_{l}^{T} \mathbf{M}_{l} \nabla \phi\left(x_{l}, y_{l}, z_{l}\right) a_{l}
$$

where, for each triangular sub-face $l, \mathbf{n}_{l}$ is the outward normal, $\mathbf{M}_{l}$ is the conductivity tensor, $\nabla \phi\left(x_{l}, y_{l}, z_{l}\right)$ is the gradient of the potential and $a_{l}$ is the scalar area. The outward normals and the area can be obtained by taking vector products of the two edges of the triangular sub-faces

$$
a_{l}=\frac{\left\|d_{1} \times d_{2}\right\|}{2} \text { and } \mathbf{n}_{l}=\frac{d_{1} \times d_{2}}{\left\|d_{1} \times d_{2}\right\|}
$$

where edges $d_{1}$ and $d_{2}$ are a pair of edges of the original hexahedron based on the centroids of the element, its faces and edges, defining a triangular surface.

Again following Penland et al. [11], the conductivity for face $l$ is defined by interpolating the conductivity of the element vertices to the centre of each triangular sub-face

$$
\mathbf{M}_{l}=\sum_{j=1}^{8} \mathbf{M}_{j} N_{j}\left(\xi_{l}, \eta_{l}, \zeta_{l}\right), \quad l=1, \ldots, 6
$$

where $\mathbf{M}_{\mathbf{j}}$ is the conductivity at each node of the element and $\left(\xi_{l}, \eta_{l}, \zeta_{l}\right)$ is the coordinate of the centroid of each triangle in the local coordinate system. Finally, the gradient of $\phi$ in $x y z$ space is determined from the gradient of $\phi$ in the natural coordinate space, via the Jacobian, as

$$
\nabla_{x y z} \phi\left(x_{l}, y_{l}, z_{l}\right)=J^{-1}\left(\xi_{l}, \eta_{l}, \zeta_{l}\right) \nabla_{\xi \eta \zeta} \phi\left(\xi_{l}, \eta_{l}, \zeta_{l}\right)
$$


where

$$
\nabla_{\xi \eta \zeta} \phi\left(\xi_{l}, \eta_{l}, \zeta_{l}\right)=\sum_{j=1}^{8} \phi_{j} \nabla_{\xi \eta \zeta} N_{j}\left(\xi_{l}, \eta_{l}, \zeta_{l}\right)
$$

with $\phi_{j}$ being the unknown potentials at the nodes. Hence

$$
\oint_{\partial B_{j, k}} \mathbf{n}^{T} \mathbf{M} \nabla \phi d S=\sum_{l=1}^{6} a_{l} \mathbf{n}_{l}^{T} \mathbf{M}_{l} \sum_{j=1}^{8} \phi_{j} J^{-1} \nabla_{\xi \eta \zeta} N_{j}\left(\xi_{l}, \eta_{l}, \zeta_{l}\right)
$$

This expression can be written in a more compact form by defining $\mathbf{u}_{k}$ to be a vector of potential values at the nodes $\phi_{j}$ of the element $E_{k}$ and $\mathbf{N}$ to be the vector of basis functions $N_{j}$, to give

$$
\oint_{\partial B_{j, k}} \mathbf{n}^{T} \mathbf{M} \nabla \phi d S=\sum_{l=1}^{6} a_{l} \mathbf{n}_{l}^{T} \mathbf{M}_{l} J^{-1} \nabla_{\xi \eta \zeta} \mathbf{N}^{T}\left(\xi_{l}, \eta_{l}, \zeta_{l}\right) \mathbf{u}_{k} .
$$

In turn, this can be simplified further by writing

$$
\oint_{\partial B_{j, k}} \mathbf{n}^{T} \mathbf{M} \nabla \phi d S=\mathbf{L}^{T} \mathbf{u}_{k}
$$

where the $j^{\text {th }}$ component of $\mathbf{L}$ is given by

$$
L_{j}=\sum_{l=1}^{6} a_{l} \mathbf{n}_{l}^{T} \mathbf{M}_{l} J^{-1} \nabla_{\xi \eta \zeta} N_{j}\left(\xi_{l}, \eta_{l}, \zeta_{l}\right)
$$

The matrix $\mathbf{L}$ is known as the diffusion coupling matrix [11].

In one sense the equations derived above are standard for the finite volume method. The key difference in the approach taken here is that for a three dimensional problem, the faces of the control volumes are subdivided into triangles. For the particular application of studying the electric potential distribution in cardiac tissue, this allows for more accurate approximation of varying fibre directions and better approximation to the flux integrals in complex geometries.

For each element $E_{k}$ in the mesh for the tissue the governing equation (4) becomes

$$
\left(\mathbf{L}_{i, k}+\mathbf{L}_{e, k}\right) \Phi_{e, k}=-\mathbf{L}_{m, k} \Phi_{m, k}
$$

where $\mathbf{L}_{i, k}$ and $\mathbf{L}_{e, k}$ are the diffusion coupling matrices on the element $E_{k}$ for the intracellular and extracellular spaces, respectively, and the potential vectors $\Phi_{e, k}$ and $\Phi_{m, k}$ contain the extracellular and 
transmembrane potentials at the vertices of element $E_{k}$. The above system of algebraic equations can be coupled together to form the global system of equations

$$
\left(\mathbf{K}_{i}+\mathbf{K}_{e}\right) \Phi_{e}=-\mathbf{K}_{i} \Phi_{m}
$$

using prolongation-like matrices [11]. The matrices $\mathbf{K}_{i}$ and $\mathbf{K}_{e}$ represent approximations to the Laplacian type operators in equations (1) and (2) also taking into account the conductivity tensors $\mathbf{M}_{i}$ and $\mathbf{M}_{e}$. It can also be shown that the discretisation of equation (3) yields

$$
\mathbf{K}_{b} \Phi_{b}=0
$$

where the matrix $\mathbf{K}_{b}$ is an approximation to the Laplacian operator in equation 3. The two equations (42) and (43) represent a system of linear equations for the unknown potentials in the extracellular space, $\Phi_{e}$, and the blood cavity, $\Phi_{b}$. Since the transmembrane potential distribution is known, the right hand side of equation (42) can be calculated via matrix-vector multiplication. Further, the system (42) and (43) can be combined into one system of equations of the form

$$
\mathrm{Ax}=\mathrm{b}
$$

where

$$
\mathbf{A}=\left(\begin{array}{c|c}
\mathbf{K}_{i}+\mathbf{K}_{e} & 0 \\
\hline 0 & \mathbf{K}_{b}
\end{array}\right), \quad \mathbf{x}=\left(\begin{array}{c}
\Phi_{e} \\
\hline \Phi_{b}
\end{array}\right), \quad \text { and } \mathbf{b}=\left(\begin{array}{c}
-\mathbf{K}_{i} \Phi_{m} \\
0
\end{array}\right)
$$

As mentioned above, the finite volume method naturally handles the Neumann type boundary conditions in an implicit fashion. This formulation also handles the continuity of potential and current at the blood-tissue interface in a natural way. To account for Dirichlet type boundary conditions at specified nodes, a row reduction method is used whereby these nodes are removed from the list of unknowns and the right hand side vector $\mathbf{b}$ adjusted accordingly. 


\subsection{Iterative Solution}

The overall matrix version of the governing equations (44) is a sparse system of linear algebraic equations. This system of equations will be solved with a generalised minimal residual algorithm, FGMRES, along with various preconditioners as implemented in the ITSOL3.0 (http://www-users.cs.umn.edu/s̃aad/software/ITSOL) package. The preconditioners used are various forms of the incomplete LU (ILU) decomposition type: (i) ILUK (ILU preconditioner with level of fill), (ii) ILUT (ILU preconditioner with threshold) and (iii) ILUC (crout version of ILUT). As will be discussed later, each geometric model has its own "best" preconditioner. The basic philosophy for solving equation (44) is to apply a preconditioner to the coefficient matrix $\mathbf{A}$ and then solve the preconditioned system with the fgmr routine from ITSOL3.0. The fgmr routine is solved iteratively until a predefined tolerance is reached. The system of equations can be solved without preconditioning, but using a preconditioner reduces the solution time. On the other hand, using a preconditioner increases the memory usage of the solution process. Hence, solving the system of equations is a trade-off between memory usage and time taken for solution.

\section{Results}

To validate the solution method discussed above, comparisons will be made with previously published analytical models of subendocardial ischaemia. Unless otherwise stated, the bidomain conductivities are those given by Clerc [28]: $\sigma_{i}^{l}=1.74 \mathrm{mS} / \mathrm{cm}, \sigma_{e}^{l}=6.25 \mathrm{mS} / \mathrm{cm}, \sigma_{i}^{t}=0.193 \mathrm{mS} / \mathrm{cm}$ and $\sigma_{e}^{t}=$ $2.36 \mathrm{mS} / \mathrm{cm}$. Blood conductivity, $\sigma_{b}$, was set at $6.7 \mathrm{mS} / \mathrm{cm}[29]$. 


\subsection{Slab Model}

For the slab model, results from the finite volume method code will be compared with those obtained from an analytical solution published previously $[13,14]$. Consider a $16 \mathrm{~cm} \times 16 \mathrm{~cm} \times 1 \mathrm{~cm}$ block of cardiac tissue with a $4 \mathrm{~cm} \times 4 \mathrm{~cm}$ region of subendocardial ischaemia located at the centre of the block. The region of ischaemia occupies $50 \%$ of the thickness ( $z$ direction) of the tissue. It was found that 61 nodes were required in each of the $x$ and $y$ directions to achieve smooth contours on the epicardial surface, especially in the region of the high potential gradients above the ischaemic boundary. This is demonstrated in Figure 6, which shows the heart surface $(z=0)$ electric potential contours for 31, 41, 51 and 61 nodes, respectively, in each of the $x$ and $y$ directions.

In the analytical model, the tissue was attached to a blood mass which extended to infinity in the positive $z$ direction. Here the blood mass extends to a depth of $25 \mathrm{~cm}$ below the region of subendocardial ischaemia and is meshed with 51 node points with increasing internodal distance as the depth increases. This number of nodes and depth of blood mass was found to be sufficient so that the epicardial potential distribution was unaffected by the position of the boundary.

It was also found that the number of node points in the tissue in the $z$ direction had little effect on the epicardial potential distribution. For models with no fibre rotation, as the number of points in the $z$ direction increased from 20 to 30 to 40 points, the relative difference in the epicardial potential compared to that with 50 points was always less than $0.1 \%$. Although 20 points across the tissue in the $z$ direction would have give an accurate solution for the purposes of this paper, all simulations were conducted with 50 points. The final model consisted of 375,821 nodes and 360,000 hexahedral finite volumes.

Figure 7 shows a comparison of the epicardial $(z=0)$ potential distributions for the analytical solution $[13,14]$ (left panel) and the finite volume solution (right panel) for a slab of cardiac tissue 
with no fibre rotation. The two contour plots are almost identical, with the main difference being the shape of the $-0.6 \mathrm{mV}$ contour line. The slight wiggles in the zero contour line in the numerical solution near the boundary of the model are simply due to clustering of nodes near the centre of the model and relative thinning near the boundary. This verifies the accuracy of the numerical model in this geometry.

\subsection{Cylindrical Model}

For this model, numerical results will be compared to the analytical results obtained using Fourier transforms and Fourier series [26]. Consider a $16 \mathrm{~cm}$ long annular cylinder of cardiac tissue of inner radius $2 \mathrm{~cm}$ and outer radius $3 \mathrm{~cm}$ with the inner core filled with blood. The ischaemic region was $4 \mathrm{~cm}$ long in the $z$-direction and covered the region $-\frac{\pi}{4} \leq \theta \leq \frac{\pi}{4}$. It again covered $50 \%$ of the tissue in the radial direction, beginning at the endocardium. For this model it was found that 30 nodes were required along the sides of the inner square of the butterfly mesh, 23 nodes radially through the thickness of the tissue and 61 nodes along the length of the cylinder ( $z$ direction) were required to achieve smooth contour lines in the region of the ischaemic boundary. Here the nodes were clustered only in the $z$ and $r$ directions. Overall, the model consisted of 445,605 nodes and 432,960 hexahedral volume elements.

Figure 8 shows a comparison of analytically and numerically obtained epicardial potential distributions for $0^{\circ}$ fibre rotation. The figure shows that there is little difference between the two distributions, again validating the numerical model. 


\subsection{Simplified Ventricular Model}

The shortcomings of the analytical models are that they only work for simple geometries (for examples, slabs, cylinders, spheres). They do not work for a general arbitrary geometry, although an analytical solution could be obtained using an ellipsoidal coordinate system in the geometry of this simplified left ventricle. In the interests of future more general applications, the finite volume method will be applied to the elliptical geometry of this ventricular model.

As mentioned above, the simplified ventricle is a half ellipsoid represented by the parametric equations [7]

$$
\begin{array}{lr}
x=a(r) \cos \theta \cos \phi & -\pi \leq \phi \leq \pi \\
y=b(r) \cos \theta \sin \phi & 0 \leq \theta \leq \frac{\pi}{2} \\
z=c(r) \sin \theta & 0 \leq r \leq 1
\end{array}
$$

where $a(r)=a_{1}+r\left(a_{2}-a_{1}\right), b(r)=b_{1}+r\left(b_{2}-b_{1}\right)$ and $c(r)=c_{1}+r\left(c_{2}-c_{1}\right)$. Here $a_{1}=1.5$, $a_{2}=2, b_{1}=1.5, b_{2}=2, c_{1}=3$ and $c_{2}=4$ define the wall of the ventricular cavity, the interior of which is filled with blood. Through the wall of the ventricle the cardiac fibres rotate linearly with the depth a total of $120^{\circ}$ anticlockwise from epicardium to endocardium. The endocardial fibres are offset $-45^{\circ}$ from the positive $z$ direction. For this mesh there were 23 nodes along the side of the inner square of the butterfly mesh and 30 nodes on the diagonals to the outer radius of the mesh. A further 50 nodes were spread across the thickness of the ventricular wall. Overall the mesh consisted of 423,975 nodes and 414,832 hexahedral elements. The region of subendocardial ischaemia covered the region $-20^{\circ} \leq \phi \leq 20^{\circ}, 50^{\circ} \leq \theta \leq 70^{\circ}$ and accounted for $50 \%$ of the thickness of the ventricular wall. Figure 9 shows an example of the mesh for the simplified ventricular model with 10 nodes along the side of the inner square and six nodes through the tissue. Note the clustering of the nodes in the radial direction through the tissue. 
Figure 10 shows the epicardial surface potential distribution for the ventricular model displayed as a polar plot. The apex of the ventricle is placed at the origin and the region of ischaemia is located on both sides of the positive $x$-axis (assumed to be horizontal and pointing to the right, away from the origin). The left hand map has no fibre rotation and in the right hand plot the fibres rotate through $120^{\circ}$ as described above. The plots show that again there is a high potential gradient over the boundary of the ischaemic region, but due to the overall geometry of the ventricle, the gradient appears only over the boundary at $\theta=70^{\circ}$. With fibre rotation included, the overall potentials are much larger and the potential gradient is a lot steeper. It is interesting to note that the region of ST depression in this model does not identify the region of subendocardial ischaemia as clearly as it does in the slab and cylindrical models. This is consistent with experimental studies [20].

It has been predicted analytically [15] that for isotropic tissue the degree of subendocardial ischaemia does not affect the epicardial potential distribution. This was also demonstrated in modelling studies on the slab [13] and cylindrical [14] geometries. Figure 11 shows epicardial potential distributions for $10 \%, 40 \%, 70 \%$ and $90 \%$ subendocardial ischaemia and there is no significant difference between the potential distributions. This confirms the original observation based on an analytical treatment of the slab geometry.

\subsection{Timings for the Iterative Solvers}

As discussed above, the system of algebraic equations arising from the finite volume method was solved using the FGMRES iterative method [30]. For this method three different preconditioners were used: ILUK, ILUT and ILUC. Each preconditioner behaved differently with each geometry considered. For each preconditioner and FGMRES solution, the maximum number of iterations was set at 600 , the Krylov subspace dimension set at 60 and the convergence tolerance for the change in size of the final to initial residuals was set at $10^{-14}$. It was found that tolerances of the order of $10^{-7}$ 
did not give consistent epicardial potential distributions, hence a very strict tolerance was chosen. The size of each model is as indicated above and there was $120^{\circ}$ fibre rotation in each case.

With each preconditioner, the level of fill, $l f i l$, governs a trade off between memory usage and computation time. The optimal solution (in the sense of least computation time) for each preconditioner and each geometry is given in Table 2. All computations were performed on a Mac Powerbook G4 with $1 \mathrm{~Gb}$ of RAM.

For ILUK, all the $l f i l$ values are small (Table 2). Increasing the $l f i l$ values increases the memory requirements for FGMRES and induces swapping within the computer memory. Consequently, this preconditioner is not recommended for these types of problems on this computer.

The ILUT preconditioner demonstrated the advantage that the level of fill was reasonably consistent across all geometries. It provided the optimum solution time for the slab geometry, but a reasonably slow solution time for the cylindrical problem.

Finally, the ILUC preconditioner gave the optimal solution times for the cylindrical and ventricular models, as well as having similar $l f i l$ values. It also showed a much greater solution time for the slab model with much larger $l f i l$ values. For the ventricular model, the ILUT and ILUC preconditioners resulted in similar solution times.

\section{Discussion and Conclusion}

This paper has presented an implementation of the finite volume method to solve the bidomain equations governing electric potential in cardiac tissue. The numerical model is tested and validated in three geometries representing the ventricular wall with subendocardial ischaemia.

The approach taken here varies slightly from previous implementations $[11,12]$ in that the computational domain is meshed in hexahedrons with each face of the hexahedral control volume broken 
down into sets of triangles. This idea allows for more accurate integration in the finite volume method and better accounts for complicated geometries and fibre rotation.

The numerical method has been validated by comparisons with previously published analytical solutions $[13,14,26]$ in terms of epicardial potential distributions. The numerical solutions show good agreement with the analytical ones when fibre rotation is ignored. Also, the semi-ellipsoidal model of the left ventricle shows that for isotropic tissue the degree of ischaemia does not affect the epicardial potential distribution, as noted in simple geometries [15].

Computation times for solving the system of algebraic equations arising from the finite volume method discretisation were also studied in detail. It was found that different preconditioners behaved differently depending on the geometry considered. This behaviour is put down to the slightly different structures of the coefficient matrix obtained for each geometry. For the slab geometry, the ILUT preconditioner is recommended, but for the cylindrical and ellipsoidal geometries, the ILUC preconditioner is recommended. It is still a matter of trial and error to obtain optimal $l f i l$ values depending on the computer architecture and size of the problem.

One clear advantage of the approach presented here is that it can handle arbitrary geometries. However, another advantage is that it would be possible to calculate the surface Laplacian [31, 32] in a straight forward manner.

Acknowledgements: The author wishes to thank Prof Craig Henriquez (Duke University) for supplying the initial finite volume code and Prof Ian Turner (Queensland University of Technology) for many valuable discussions regarding the finite volume method. 


\section{References}

[1] Schmitt, O. H. Biological information processing using the concept of interpenetrating domains In Leibovic, K. N., editor, Information Processing in the Nervous System, chapter 18, pages 325-331 Springer-Verlag, New York, 1969.

[2] Tung, L. A Bi-domain model for describing ischaemic myocardial D-C potentials $\mathrm{PhD}$ thesis, Massachusetts Institute of Technology, June 1978.

[3] Miller, W. T. and Geselowitz, D. B. Simulation studies of the electrocardiogram I: The normal heart Circulation Research, 43:301-315, 1978.

[4] Pollard, A. E., Hooke, N., and Henriquez, C. S. Cardiac propagation simultion Crit. Rev. Biomed. Eng, 20:171-210, 1992.

[5] Buist, M., Sands, G., Hunter, P., and Pullan, A. A deformable finite element derived finte difference method for cardiac activation problems Annals of Biomedical Engineering, 31:577588, 2003.

[6] Trew, M. L., Smaill, B. H., Bullivant, D. P., Hunter, P. J., and Pullan, A. J. A generalised finite difference method for modelling cardiac electrical activation on arbitrary, irregular computational meshes Mathematical Biosciences, 198:169-189, 2005.

[7] Colli-Franzone, P., Guerri, L., and Taccardi, B. Modeling ventricular excitation: axial and orthotropic anisotropy effects on wavefronts and potentials Mathematical Biosciences, 188(12):191-205, 2004. 
[8] Colli Franzone, P., Pavarino, L. F., and Taccardi, B. Effects of transmural electrical heterogeneities and electrotonic interactions on the dispersion of cardiac repolarization and action potential duration: A simulation study Mathematical Biosciences, 204(1):132-165, 2006.

[9] Sundnes, J., Nielsen, B. F., Mardal, K. A., Cai, X., Lines, G. T., and Tveito, A. On the computational complexity of the bidomain and the monodomain models of electrophysiology Annals of Biomedical Engineering, 34(7):1088-1097, 2006.

[10] MacLachlan, M. C., Sundnes, J., Skavhaug, O., Lysaker, M., Nielsen, B. F., and Tveito, A. A linear system of partial differential equations modeling the resting potential of a heart with regional ischemia Mathematical Biosciences, 210(1):238-252, 2007.

[11] Penland, R. C., Harrild, D. M., and Henriquez, C. S. Modeling impulse propagation and extracellular potential distributions in anisotropic cardiac tissue using a finite volume element discretization Computing and Visualization in Science, 4(4):215-226, 2002.

[12] Trew, M., Le Grice, I., Smaill, B., and Pullan, A. A finite volume method for modeling discontinuous electrical activation in cardiac tissue Annals of Biomedical Engineering, 33(5):590-602, 2005.

[13] Johnston, P. R., Kilpatrick, D., and Li, C. Y. The importance of anisotropy in modelling ST segment shift in subendocardial ischaemia IEEE Transactions on Biomedical Engineering, 48(12):1366-1376, December 2001.

[14] Johnston, P. R. and Kilpatrick, D. The effect of conductivity values on ST segment shift in subendocardial ischaemia IEEE Transactions on Biomedical Engineering, 50(2):150-158, February 2003. 
[15] Johnston, P. R. The effect of simplifying assumptions in the bidomain model of cardiac tissue: Application to ST-Segment Shifts During Partial Ischaemia Mathematical Biosciences, 198(1):97-118, 2005.

[16] Gulrajani, R. M. Bioelectricity and Biomagnetism John Wiley and Sons, 1998.

[17] Hopenfeld, B., Stinstra, J. G., and MacLeod, R. S. The effect of conductivity on st-segment epicardial potentials arising from subendocardial ischemia Annals of Biomedical Engineering, 33(6):751-763, 062005.

[18] Rodriguez, B., Trayanova, N., and Noble, D. Modelling cardiac ischaemia Ann. N.Y. Acad. Sci., 1080:395-414, 2006.

[19] Kléber, A. G., Janse, M. J., van Capelle, F. J. L., and Durrer, D. Mechanism and time course of S-T and T-Q segment changes during acute regional myocardial ischemia in the pig heart determined by extracellular and intracellular recordings Circ. Res., 42(5):603-613, 1978.

[20] Li, D., Li, C. Y., Yong, A. C., and Kilpatrick, D. Source of electrocardiographic ST changes in subendocardial ischemia Circulation Research, 82:957-970, 1998.

[21] Potse, M., Coronel, R., Falcao, S., LeBlanc, A. R., and Vinet, A. The effect of lesion size and tissue remodeling on ST deviation in partial-thickness ischemia; Heart Rhythm, 4(2):200-206, 2007.

[22] Roth, B. J. Action potential propagation in a thick strand of cardiac muscle Circulation Research, 68(1):162-173, January 1991. 
[23] Hopenfeld, B., Stinstra, J. G., and Mac $\{$ L $\}$ eod, R. S. Mechanism for $\{$ ST $\}$ depression associated with contiguous subendocardial ischaemia Journal of Cardiovascular Electrophysiology, 15:1200-1206, 2004.

[24] Krassowska, W. and Neu, J. Effective boundary conditions for syncytial tissues IEEE Transactions on Biomedial Engineering, 41(2):143-150, 1994.

[25] LeGrice, I. J., Smaill, B. H., Chai, L. Z., Edgar, S. G., Gavin, J. B., and Hunter, P. J. Laminar structure of the heart: ventricular myocyte arrangement and connective tissue architecture in the dog Am. J. Physiol., 269:H571-H582, 1995.

[26] Johnston, P. R. A cylindrical model for studying subendocardial ischaemia in the left ventricle Mathematical Biosciences, 186(1):43-61, 2003.

[27] Johnston, P. R. and Elliott, D. A sinh transformation for evaluating nearly singular boundary element integrals International Journal for Numerical Methods in Engineering, 62(4):564-578, 2005.

[28] Clerc, L. Directional differences of impulse spread in trabecular muscle from mammalian heart Journal of Physiology, 255:335-346, 1976.

[29] Rush, S., Abildskov, J. A., and McFee, R. Resistivity of body tissues at low frequencies Circulation Research, 12:40-50, 1963.

[30] Saad, Y. Iterative Methods for Sparse Linear Systems SIAM, second edition, 2003.

[31] Johnston, P. R. The potential for Laplacian maps to solve the inverse problem of electrocardiography IEEE Trans. Biomed. Engng, 43(4):384-393, April 1996. 
[32] Johnston, P. R. The Laplacian inverse problem of electrocardiography: An eccentric spheres study IEEE Trans. Biomed. Engng, 44(7):539-548, 1997. 


\section{Figure and Table Captions}

Figure 1: Plot of the function $\Psi(t)$ (equation (16)) describing the transition from ischaemic tissue to normal tissue for two values of the parameter $\lambda_{t}$.

Figure 2: Plot of the node clustering function (equation (17)) demonstrating the clustering around the point $s=0$ (ie with $a=0$ ) for three different values of the parameter $b$.

Figure 3: Depiction of the abstract "butterfly" mesh and the resulting end mesh (b) for the cylindrical model with 23 nodes along the sides of the inner square and 30 nodes in the radial direction through the ventricular tissue. Note the clustering of the nodes midway through the tissue, corresponding to the top of the ischaemic region.

Figure 4: The Jacobian mapping from an arbitrary hexahedron to a cube.

Figure 5: Representation of the hexahedral element $E_{k}$ attached to the node $P_{j}$ (obscured by the shaded inner box and numbered node 1 in the local $(\xi, \eta, \zeta)$ coordinate system (Figure 4$)$ ). The three shaded quadrilateral surfaces form the surface of the control volume $B_{j}$ surrounding the node $P_{j}$ within the element $E_{k}$, that is, the sub-box $B_{j, k}$. The shaded surfaces then present $\partial B_{j, k}$. Here, each shaded quadrilateral surface is divided into two triangular sub-faces, of which one is shown with normals $n_{1}$ and $n_{2}$. The vectors $d_{1}$ and $d_{2}$, shown in the figure, give rise to the normal $n_{1}$. (Another pair of vectors using the opposite edges of the same quadrilateral face would give rise the normal $n_{2}$.) The point $c$ is the centroid of the volume element, the points $f$ represent centroids of the three relevant faces of the element and the points $e$ represent the centroids of the relevant edges of the element.

Figure 6: Comparison of heart surface $(z=0)$ electric potential distributions for various numbers of nodes in the $x$ and $y$ direction for the slab model.

Figure 7: Epicardial potential distribution in millivolts on the surface $(z=0)$ of the slab model with no fibre rotation for the (a) analytical and (b) numerical solutions. The solid thick line is the zero 
potential line and the dashed lines are contours of negative potential. The contour interval is $0.2 \mathrm{mV}$.

Figure 8: Epicardial potential distribution in millivolts on the surface of the cylindrical model with no fibre rotation for the (a) analytical and (b) numerical solutions. The surface is the unfolded surface of the cylinder, cut along the $z$ direction at $\theta=\pi$. The format is the same as in Figure 7 with the additional solid lines representing positive potentials.

Figure 9: An example of the mesh for the simplified ventricular model with 10 nodes across the inner square and 6 nodes radially through the ventricular tissue. Note that simulations were performed on a mesh with 23 nodes across the inner square and 30 nodes radially. The coordinate axes show the orientation of the model.

Figure 10: Epicardial potential distribution in millivolts on the surface of the ellipsoidal ventricular model with both no fibre rotation and $120^{\circ}$ fibre rotation. The surface is the flattened surface of the ellipsoid with the apex of the ventricle placed at the origin of the polar plot, with the axes referring to the global coordinate system. The format is the same as in Figure 7.

Figure 11: Epicardial potential distribution in millivolts on the surface of the isotropic ellipsoidal ventricular model with varying degrees of subendocardial ischaemia. The format is the same as in Figure 7.

Table 1: Coefficients for the basis functions used in mapping of an arbitrary hexahedron into a cube.

Table 2: Comparison of solution times in each of the three geometries considered and the three preconditioners used. 


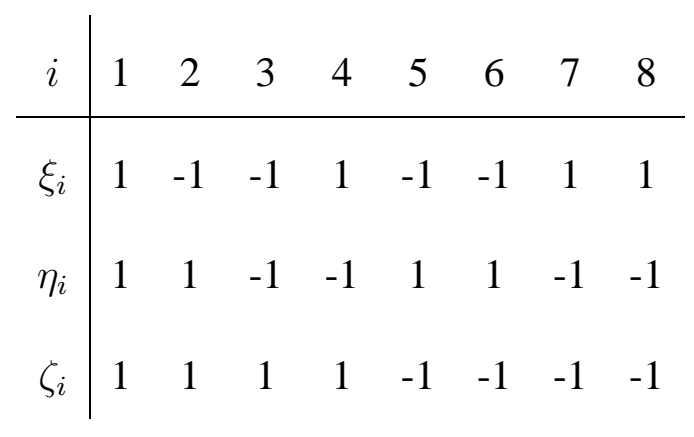

Table 1: 


\begin{tabular}{c|c|c|c|c|c}
\multirow{2}{*}{ Preconditioner } & Geometry & lfil & Preconditioning & FGMRES & Total Solution \\
& Slab & 1 & 14.42 & 205 & 411 \\
& Cylinder & 0 & 6.58 & 600 & 1140 \\
\hline \multirow{2}{*}{ ILUT } & Ventricular & 1 & 20.58 & 295 & 704 \\
& Slab & 24 & 31.77 & 121 & 248 \\
& Cylinder & 21 & 61.57 & 472 & 1007 \\
& Ventricular & 24 & 83.34 & 137 & 349 \\
\hline \multirow{2}{*}{ ILUC } & Slab & 54 & 31.41 & 433 & 1076 \\
& Cylinder & 39 & 26.66 & 252 & 650 \\
& Ventricular & 34 & 21.90 & 137 & 324
\end{tabular}

Table 2: 


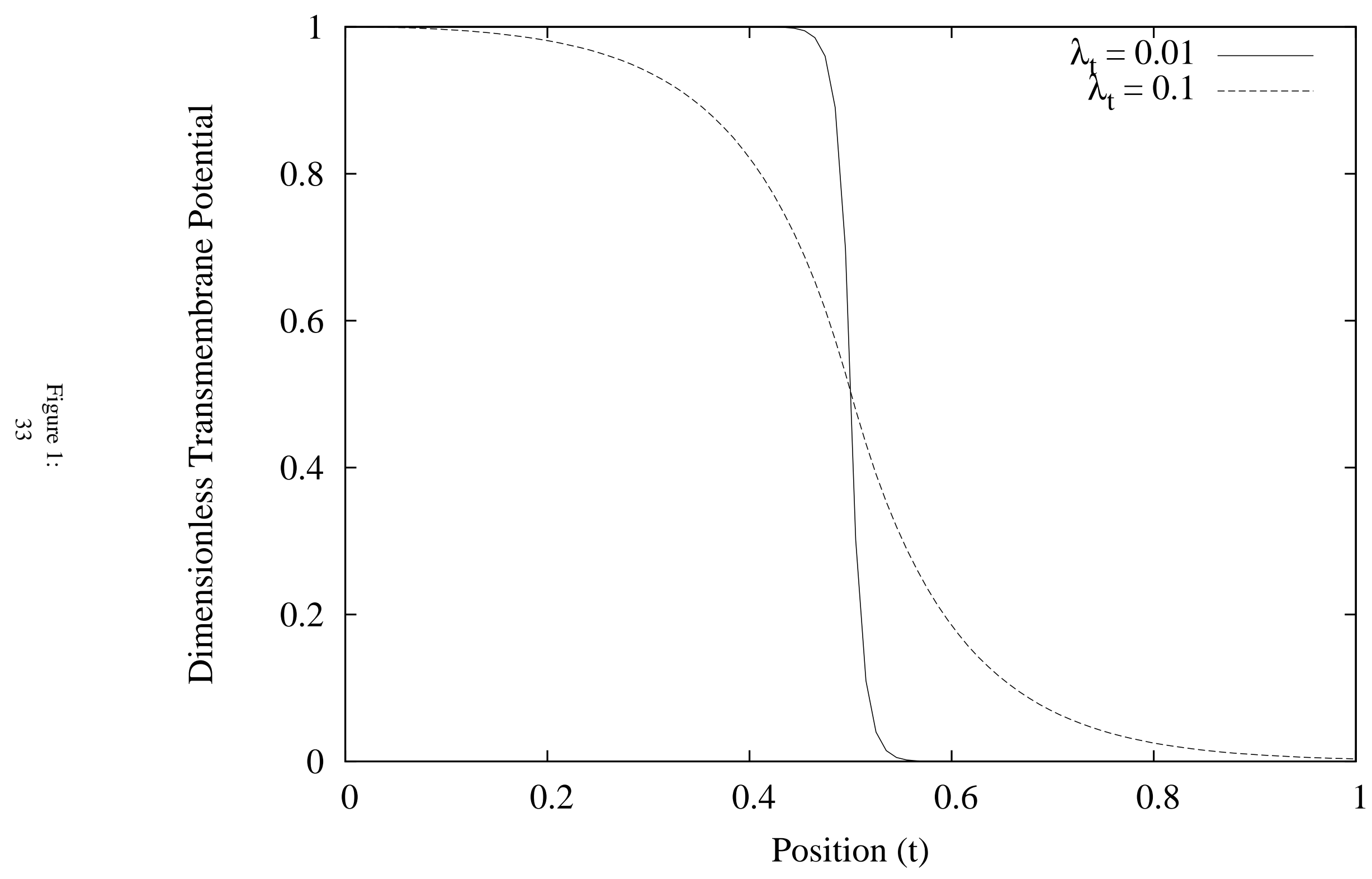




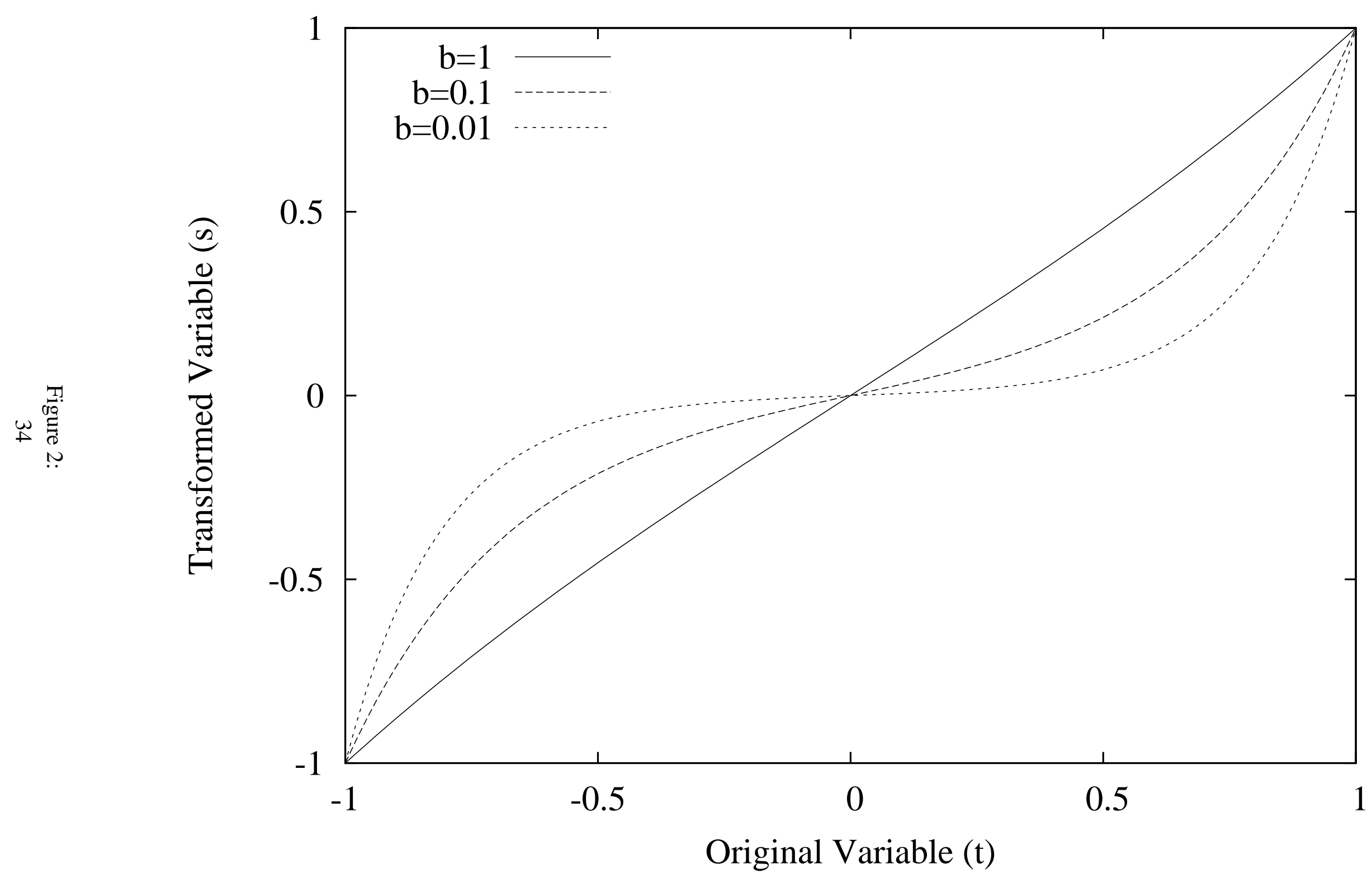




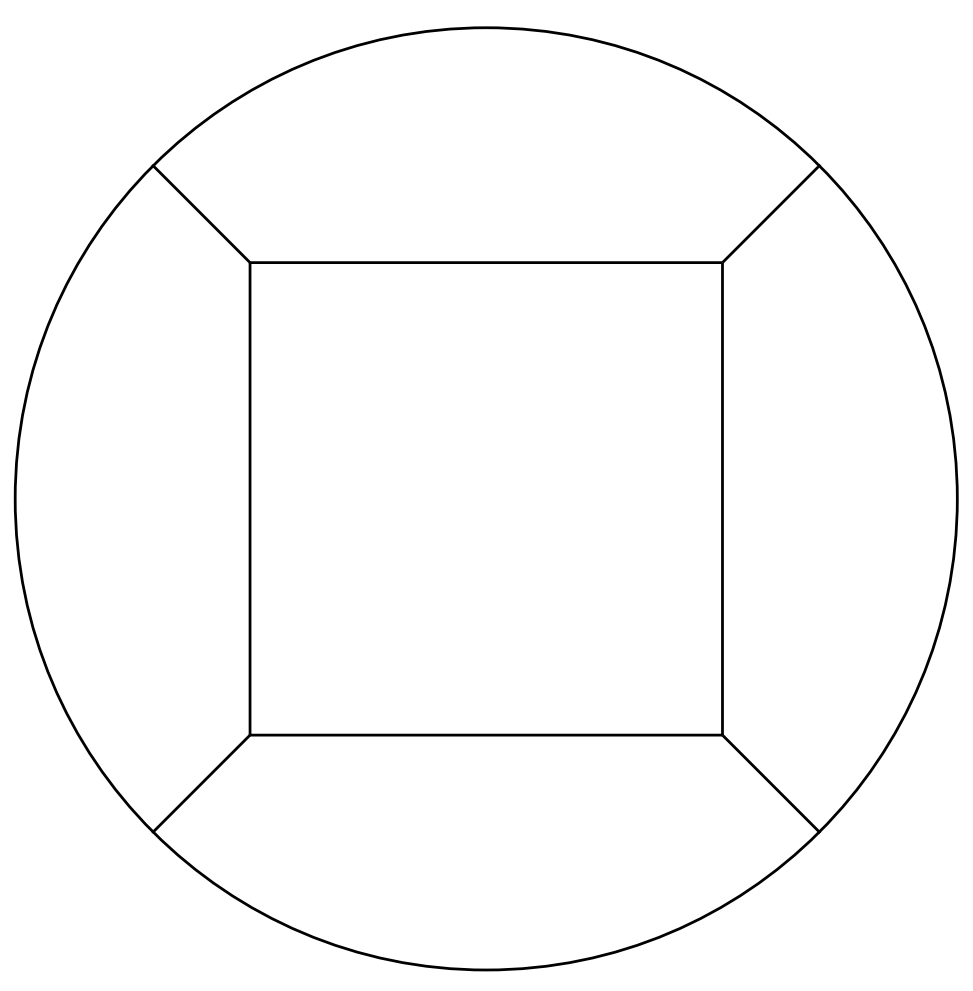

(a) "Butterfly" Mesh

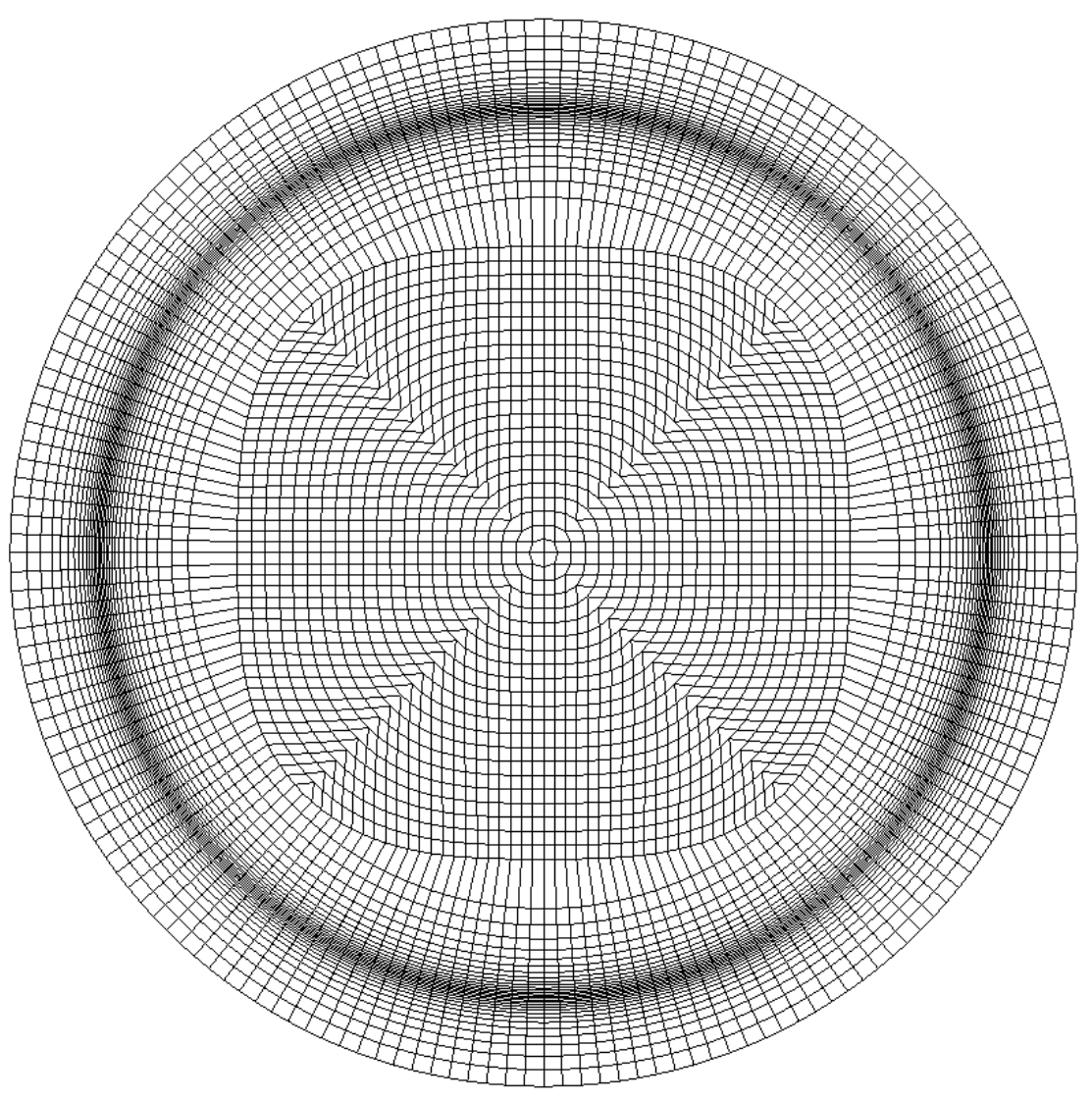

(b) Computational mesh

Figure 3: 


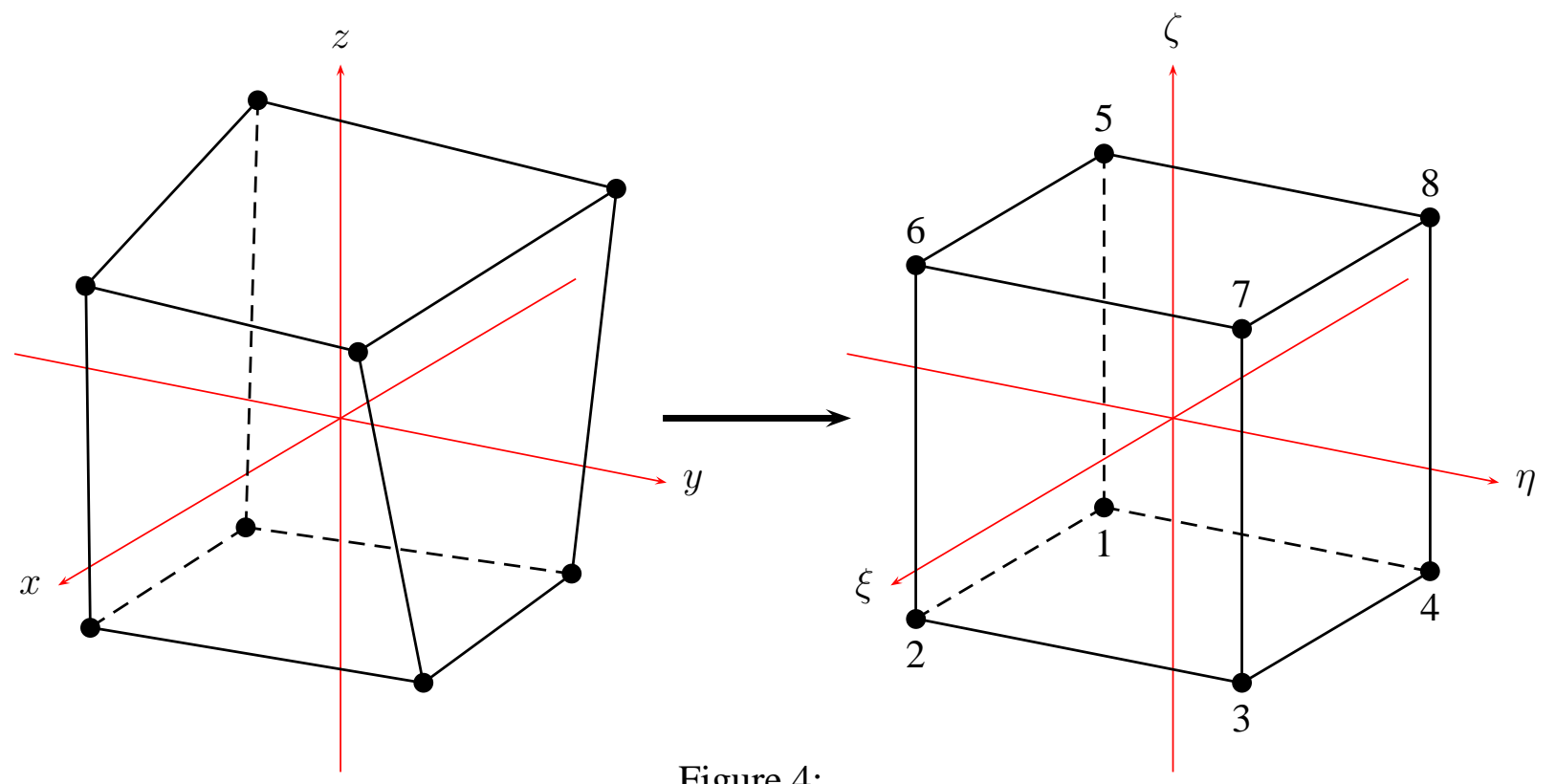

Figure 4: 


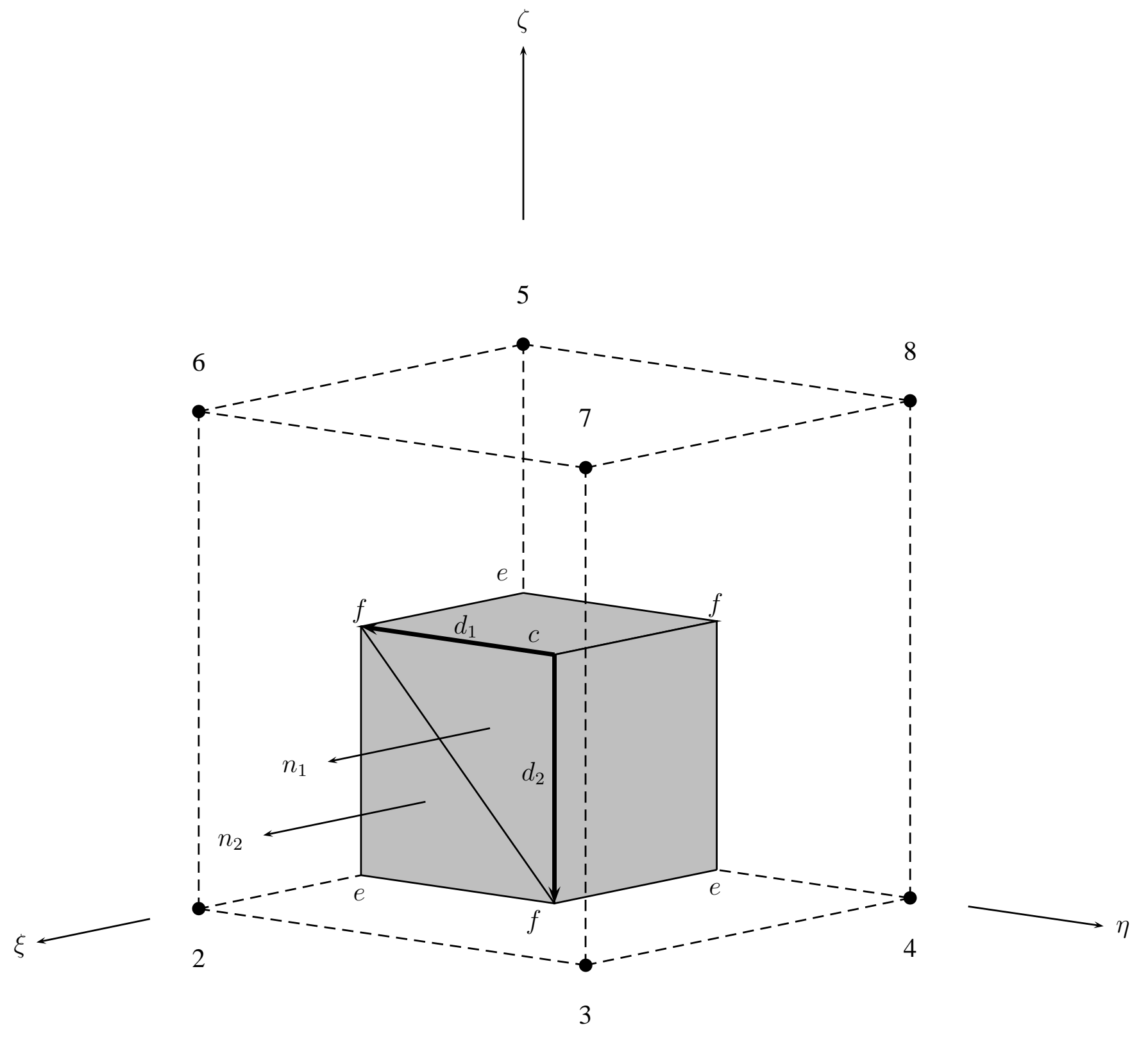

Figure 5: 
Epicardial Potential Distribution (mV)

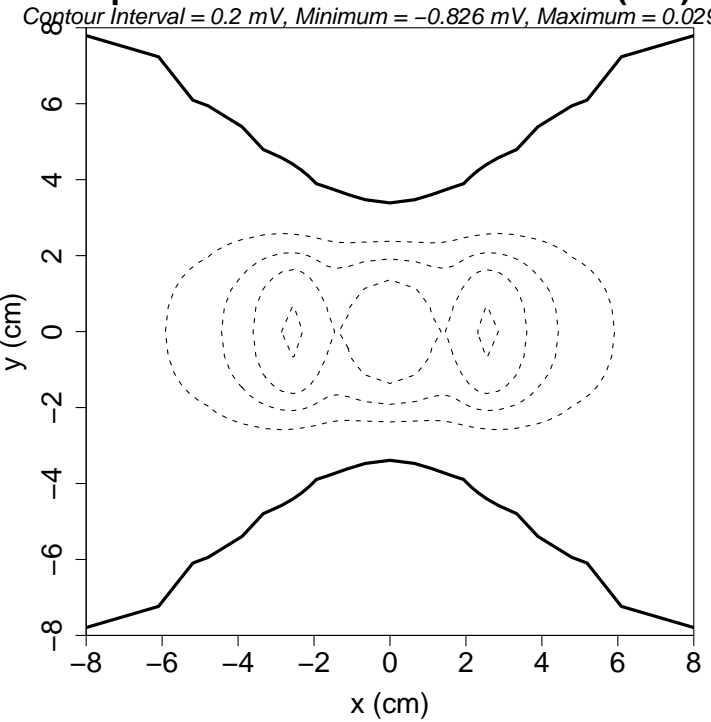

(a) Nodes in $x$ and $y$ directions: 31

Epicardial Potential Distribution ( $\mathrm{mV}$ )

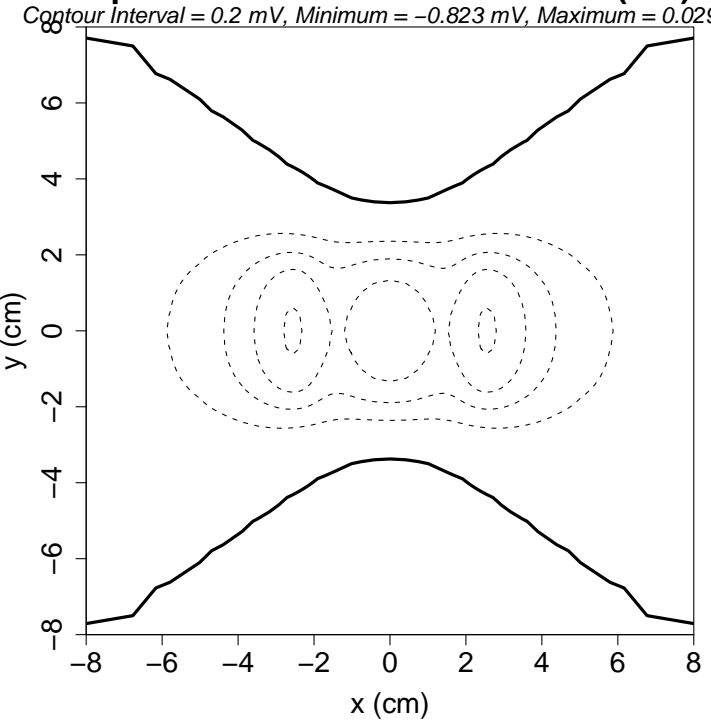

(c) Nodes in $x$ and $y$ directions: 51
Epicardial Potential Distribution ( $\mathrm{mV}$ )

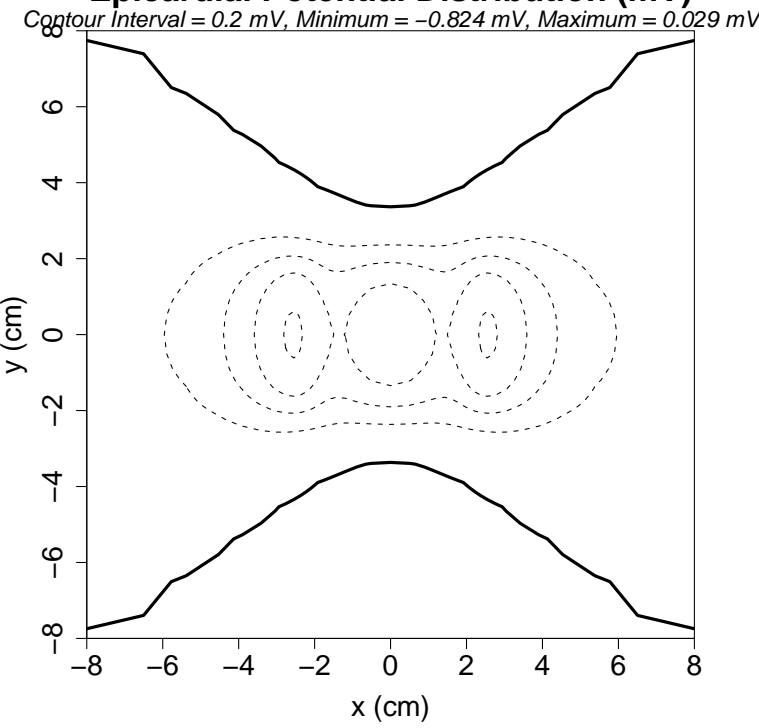

(b) Nodes in $x$ and $y$ directions: 41

Epicardial Potential Distribution (mV)

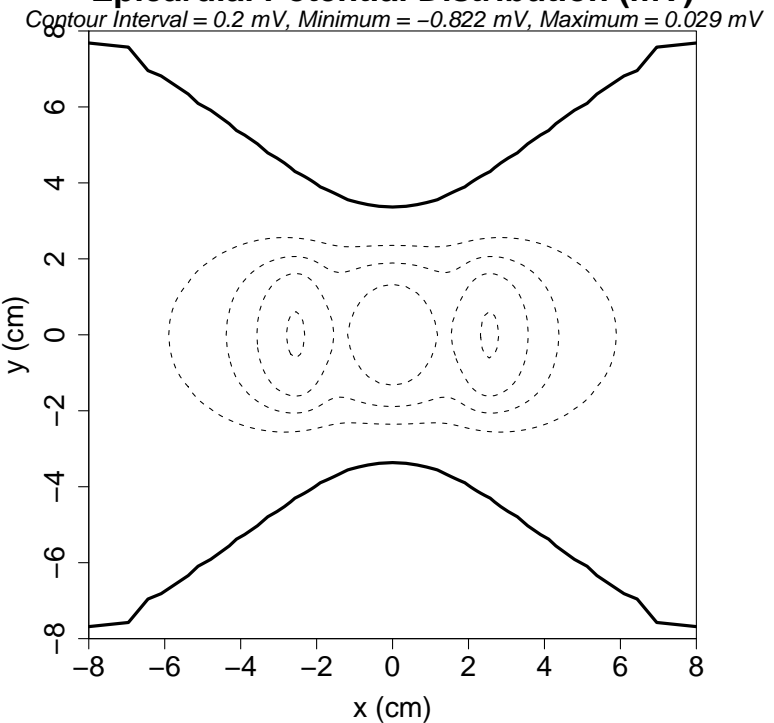

(d) Nodes in $x$ and $y$ directions: 61

Figure 6: 


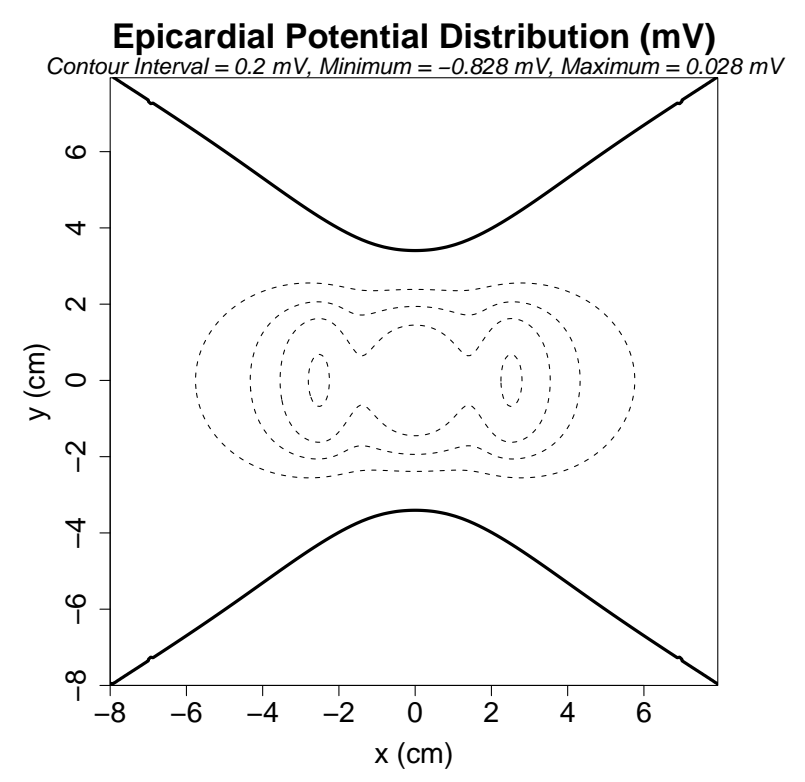

(a) Analytical Solution

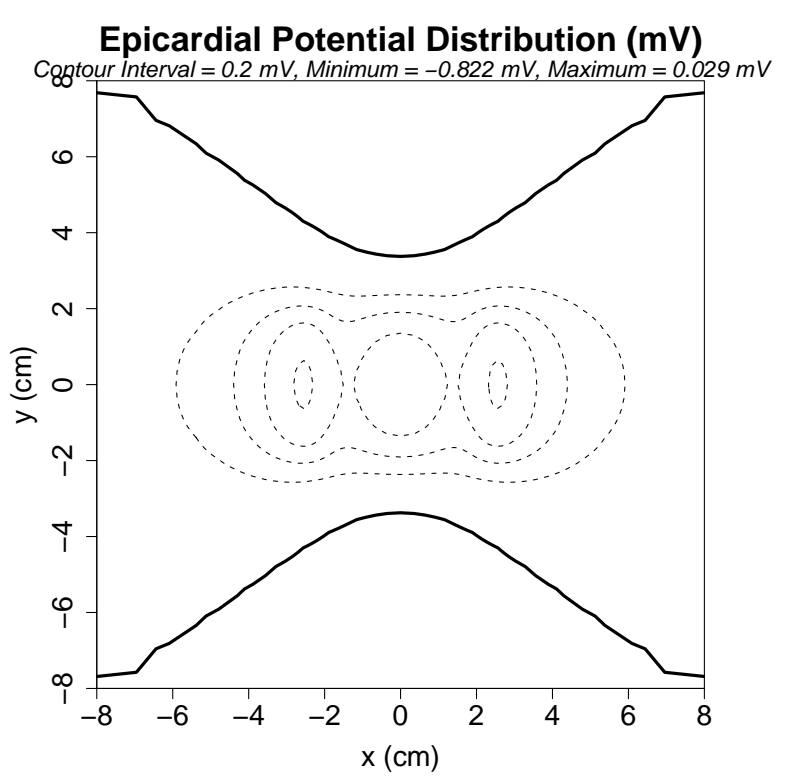

(b) Numerical Solution

Figure 7: 


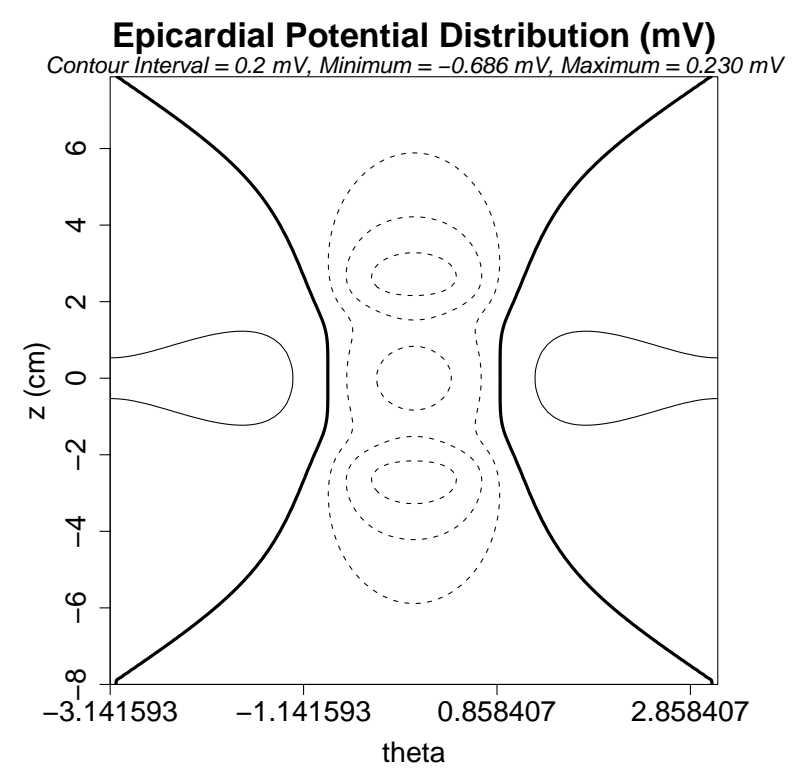

(a) Analytical Solution

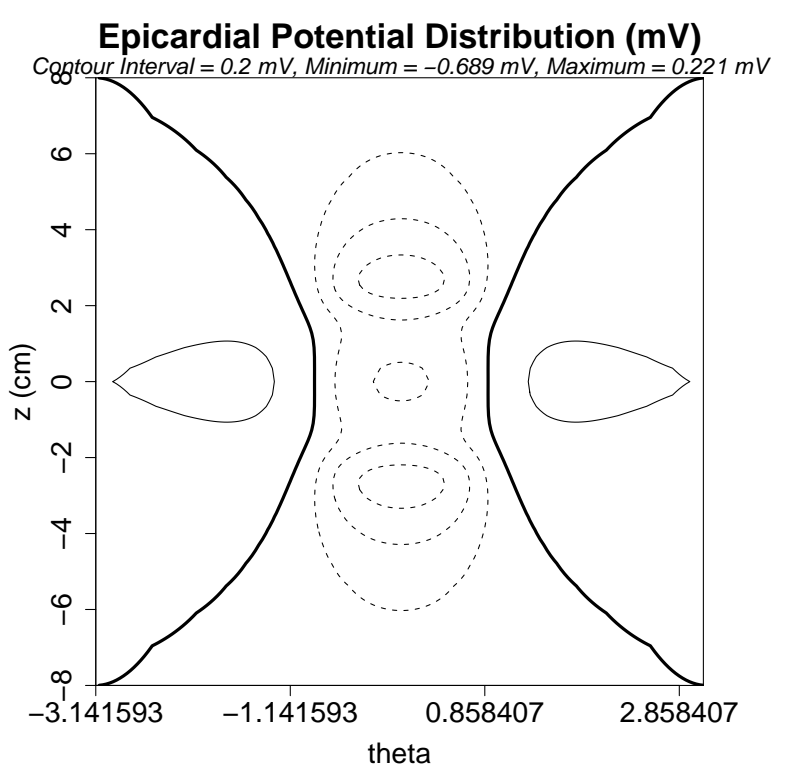

(b) Numerical Solution

Figure 8: 


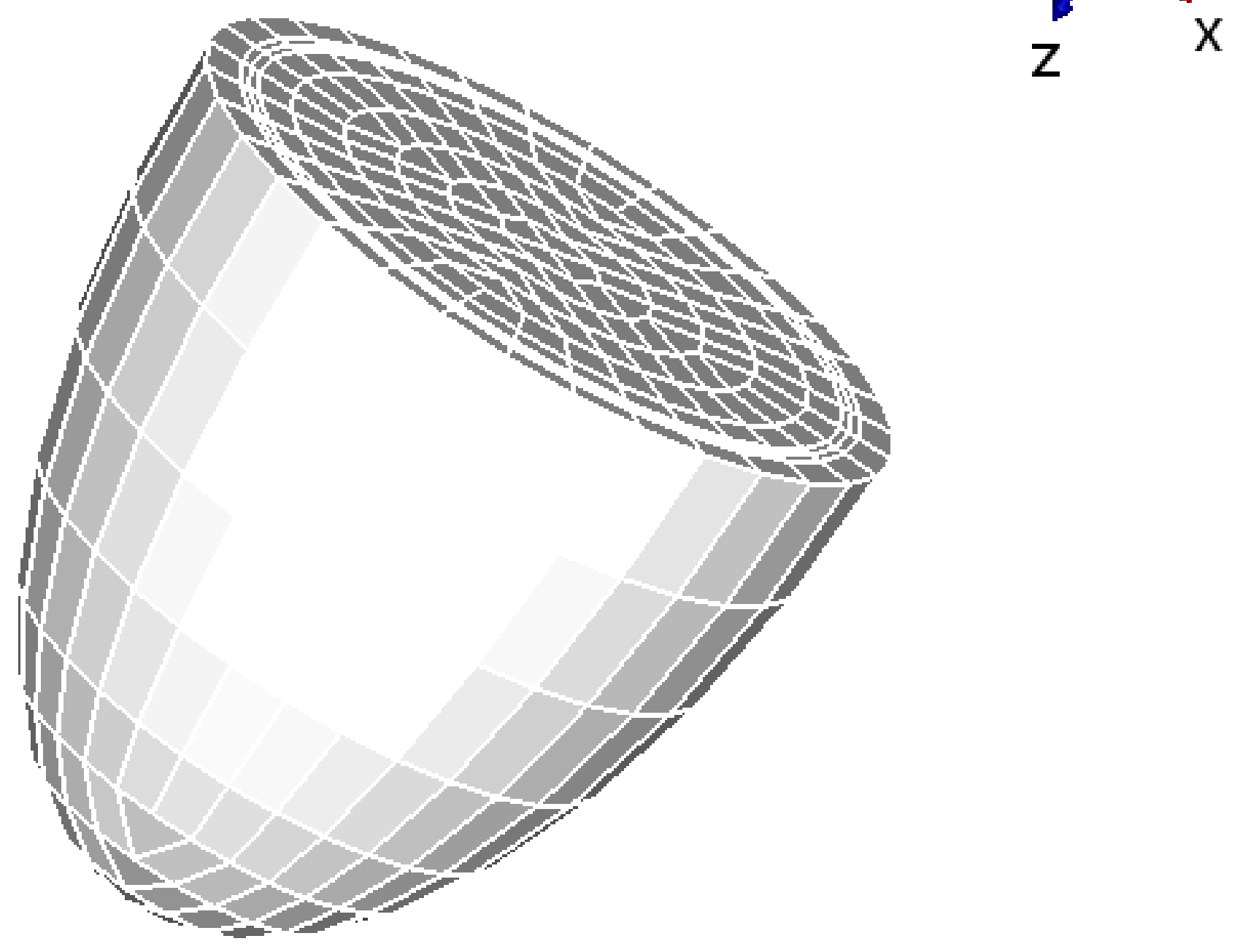

Figure 9: 


\section{Epicardial Potential Distribution ( $\mathrm{mV}$ )}

Contour Interval $=0.2 \mathrm{mV}$, Minimum $=-1.075 \mathrm{mV}$, Maximum $=0.156 \mathrm{mV}$

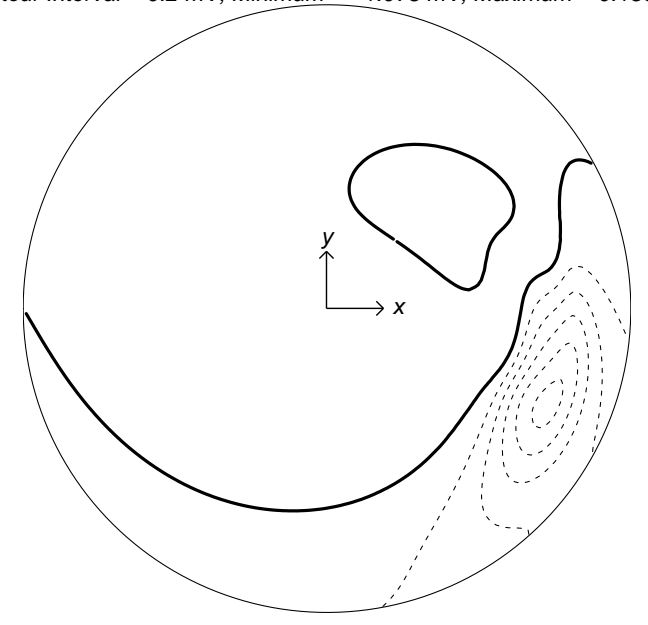

\section{Epicardial Potential Distribution (mV)}

Contour Interval $=0.2 \mathrm{mV}$, Minimum $=-1.173 \mathrm{mV}$, Maximum $=0.353 \mathrm{mV}$

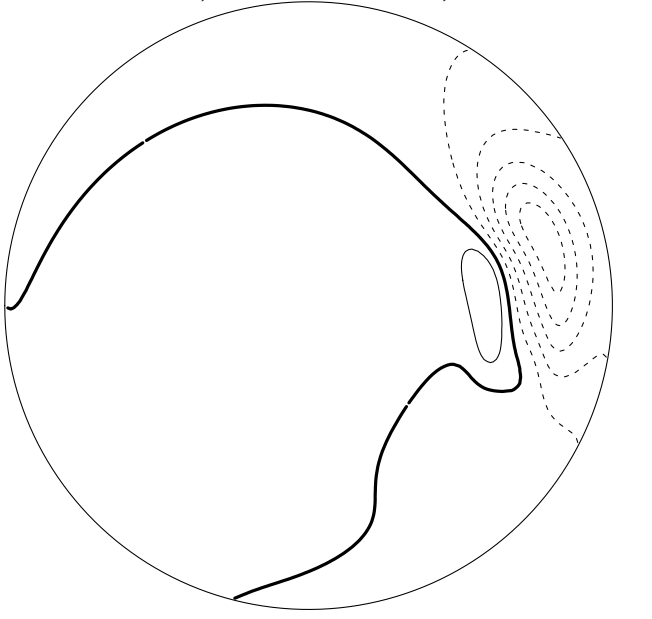

(a) No fibre rotation

(b) $120^{\circ}$ fibre rotation

Figure 10: 
Epicardial Potential Distribution ( $m$ V) Contour Interval $=0.2 \mathrm{mV}$, Minimum $=-5.063 \mathrm{mV}$, Maximum $=0.001 \mathrm{mV}$

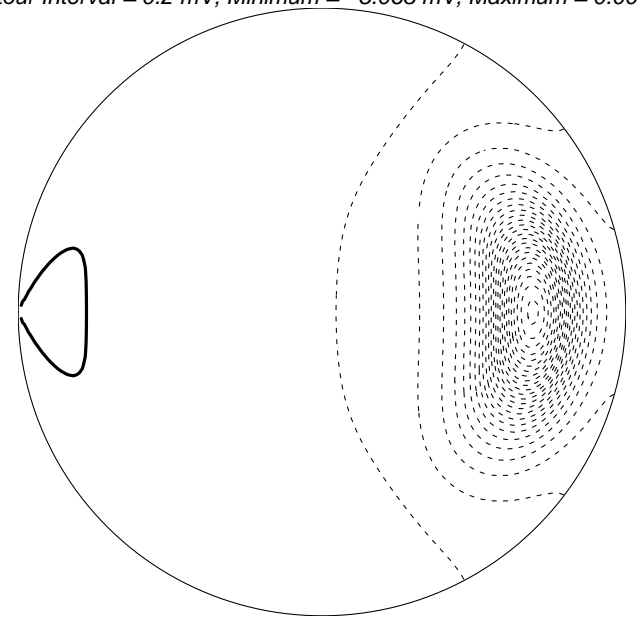

(a) $10 \%$ Subendocardial Ischaemia

Epicardial Potential Distribution ( $m$ V) Contour Interval $=0.2 \mathrm{mV}$, Minimum $=-5.063 \mathrm{mV}$, Maximum $=0.001 \mathrm{mV}$

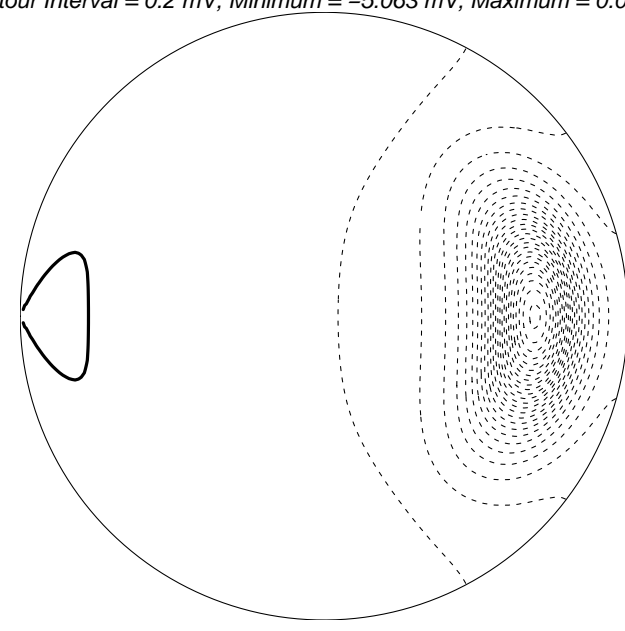

(a) $70 \%$ Subendocardial Ischaemia
Epicardial Potential Distribution (mV) Contour Interval $=0.2 \mathrm{mV}$, Minimum $=-5.063 \mathrm{mV}$, Maximum $=0.001 \mathrm{mV}$

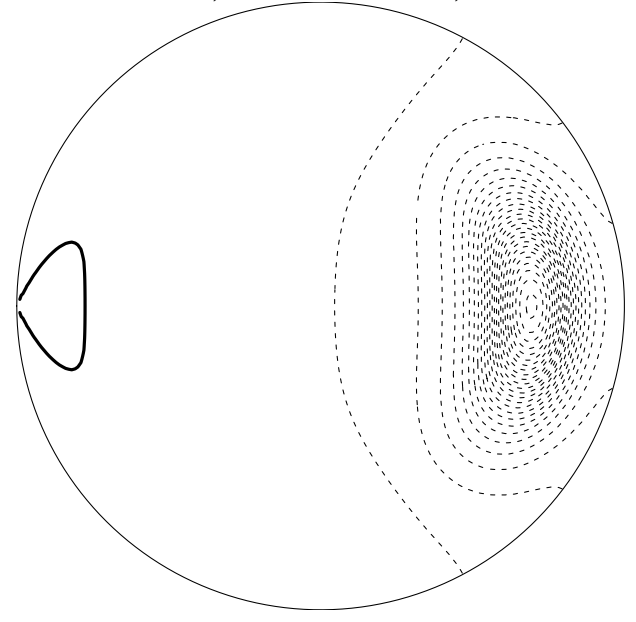

(b) $40 \%$ Subendocardial Ischaemia

Epicardial Potential Distribution ( $\mathrm{mV}$ ) Contour Interval $=0.2 \mathrm{mV}$, Minimum $=-5.063 \mathrm{mV}$, Maximum $=0.001 \mathrm{mV}$

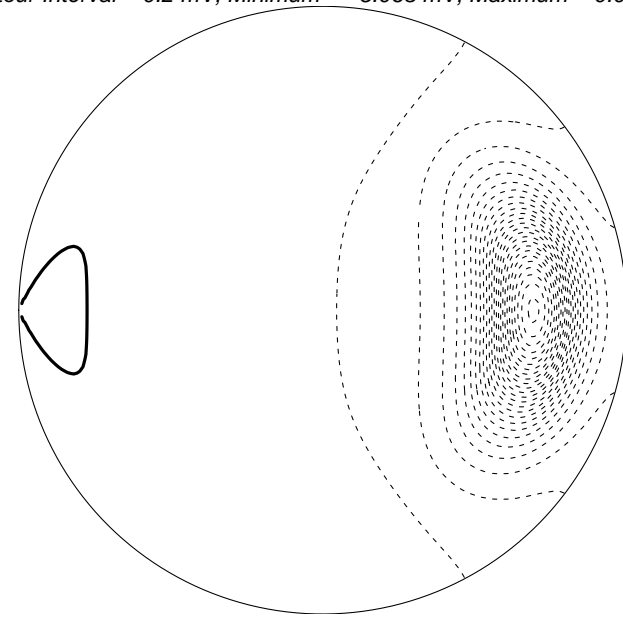

(b) $90 \%$ Subendocardial Ischaemia

Figure 11: 